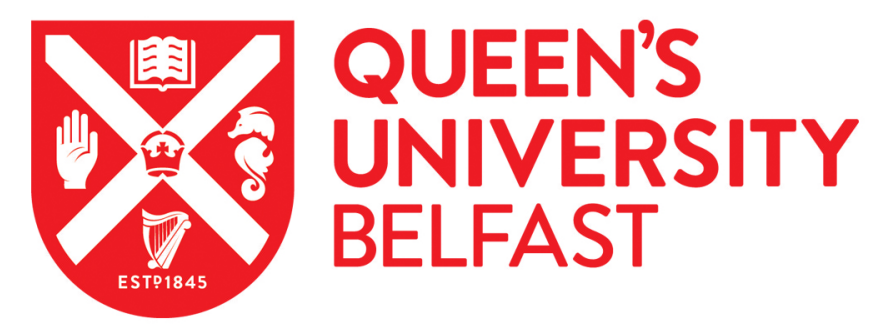

\title{
Direct correlation between potentiometric and impedance biosensing of antibody-antigen interactions using an integrated system
}

Tsai, M-Y., Creedon, N., Brightbill, E., Pavlidis, S., Brown, B., Gray, D., Shields, N., Sayers, R., Mooney, M., O'Riordan, A., \& Vogel, E. (2017). Direct correlation between potentiometric and impedance biosensing of antibody-antigen interactions using an integrated system. Applied Physics Letters, 111(7), 1-6. https://doi.org/10.1063/1.4986190

Published in:

Applied Physics Letters

Document Version:

Peer reviewed version

Queen's University Belfast - Research Portal:

Link to publication record in Queen's University Belfast Research Portal

Publisher rights

Copyright 2017 AIP.

This work is made available online in accordance with the publisher's policies. Please refer to any applicable terms of use of the publisher.

\section{General rights}

Copyright for the publications made accessible via the Queen's University Belfast Research Portal is retained by the author(s) and / or other copyright owners and it is a condition of accessing these publications that users recognise and abide by the legal requirements associated with these rights.

Take down policy

The Research Portal is Queen's institutional repository that provides access to Queen's research output. Every effort has been made to ensure that content in the Research Portal does not infringe any person's rights, or applicable UK laws. If you discover content in the Research Portal that you believe breaches copyright or violates any law, please contact openaccess@qub.ac.uk. 
1 Direct Correlation between Potentiometric and Impedance Biosensing of Antibody-Antigen Interactions using an Integrated System

4 Meng-Yen Tsai,,${ }^{1, *}$ Niamh Creedon, ${ }^{2, \dagger}$ Eleanor Brightbill,,${ }^{1, \dagger}$ Spyridon Pavlidis, ${ }^{1, \dagger}$ Billyde Brown, ${ }^{3}$ Darren 5 W. Gray, ${ }^{4}$ Niall Shields, ${ }^{4}$ Ríona Sayers, ${ }^{5}$ Mark H. Mooney, ${ }^{4}$ Alan O'Riordan ${ }^{2}$ and Eric M. Vogel ${ }^{1, *}$

$7 \quad{ }^{1}$ School of Materials Science and Engineering, Georgia Institute of Technology, Atlanta, GA 30332, USA

$8 \quad{ }^{2}$ Nanotechnology Group, Tyndall National Institute, University College Cork, Cork, T12R5CP, Ireland

$9{ }^{3}$ Georgia Tech Manufacturing Institute, Georgia Institute of Technology, Atlanta, GA 30332, USA

$10{ }^{4}$ Institute for Global Food Security, School of Biological Sciences, Queen's University Belfast, Belfast, 11 Northern Ireland, BT9 5AG, United Kingdom

$12{ }^{5}$ Animal and Bioscience Research Department, Animal and Grassland Research and Innovation Centre, 13 Teagasc, Moorepark, Fermoy, Co. Cork P61 C996, Ireland

$14 *$ corresponding authors

15 †These authors contribute equally to this work

16 M.-Y. Tsai: mytsai@ gatech.edu

17 E. M. Vogel: eric.vogel@mse.gatech.edu 


\section{Abstract}

2 A fully integrated system that combines extended gate field-effect transistor (EGFET)-based

3 potentiometric biosensors and electrochemical impedance spectroscopy (EIS)-based biosensors has been

4 demonstrated. This integrated configuration enables the sequential measurement of the same

5 immunological binding event on the same sensing surface, and consequently sheds light on the

6 fundamental origins of sensing signals produced by FET and EIS biosensors, as well as the correlation

7 between the two. Detection of both BSA/anti-BSA model system in buffer solution and bovine

8 parainfluenza antibodies in complex blood plasma samples were demonstrated using the integrated

9 biosensors. Comparison of the EGFET and EIS sensor responses reveals similar dynamic ranges, while

10 equivalent circuit modeling of the EIS response shows that the commonly reported total impedance

11 change $\left(\Delta Z_{\text {total }}\right)$ is dominated by the change in charge transfer resistance $\left(R_{\mathrm{ct}}\right)$ rather than surface

12 capacitance $\left(C_{\text {surface }}\right)$. Using electrochemical kinetics and the Butler-Volmer equation, we unveil that the

13 surface potential and charge transfer resistance, measured by potentiometric and impedance biosensors,

14 respectively, are, in fact, intrinsically linked. This observation suggests that there is no significant gain in

15 using FET/EIS integrated system and leads to the demonstration that low-cost EGFET biosensors are 16 sufficient as a detection tool to resolve the charge information of biomolecules for practical sensing 17 applications.

Text

20 The ability to detect biochemical species rapidly without complicated sample preparations in a centralized

21 laboratory is essential for medical care, disease diagnosis, food safety and environmental monitoring.

22 Optical biosensing that requires secondary markers to label target biomolecules (e.g., fluorescence

23 spectroscopy) is time consuming in sample preparation and response. ${ }^{1}$ In contrast, label-free electrical

24 biosensors are particularly promising as point-of-care sensor devices since they can be miniaturized 
1 through cost-effective microfabrication. Relying directly on the immunological affinity between

2 immobilized surface probes and targets in an analyte solution, the resulting change of the surface

3 properties is amplified and converted to an electrical output signal by a transducer. Potentiometric and

4 impedance biosensors are two of the most common transducers for label-free biosensing. Potentiometric

5 biosensors detect the change of surface potential under DC operation due to the attachment of charged

6 target biomolecules to the probes on the sensing surface, and are most commonly implemented in the

7 form of ion-sensitive FETs (ISFETs) or in a similar but a simplified configuration - extended gate FETs

8 (EGFETs). ${ }^{2-5}$ Alternatively, impedance biosensors use electrochemical impedance spectroscopy (EIS) to

9 measure the change of electrical current and, in turn, the electrical impedance of a biological interface

10 under DC bias and $\mathrm{AC}$ oscillation. ${ }^{6-9}$ Cost-effective potentiometric and impedance sensors are capable of

11 label-free operation that both simplifies the sample preparation steps and enable on-site detection with

12 shorter turnaround time.

13 Although various nanomaterial-based ISFETs have been used for potentiometric biosensors including 14 silicon nanowires, ${ }^{10}$ graphene, ${ }^{11}$ carbon nanotubes, ${ }^{12}$ we have reported that the sensitivity of a 15 potentiometric biosensor is independent of the choice of the transducer, and instead relies only on the 16 sensing surface. ${ }^{4}$ Moreover, ISFETs require a sophisticated encapsulation scheme to protect the 17 semiconductor device from exposure to the liquid environment that can hamper reliability and stability. ${ }^{13}$

18 Consequently, if either the sensing surface or the readout component fails, the entire ISFET sensor 19 becomes unusable. EGFET biosensors, by contrast, provide a simpler and a more robust design for liquid 20 phase sensing by separating the sensing chip (an extended gate) from the readout transducer (FET), and

21 the sensitivity is comparable to the conventional ISFET. ${ }^{4}$ Moreover, the EGFET is a promising tool for

22 disease diagnosis with a comparable performance to ELISA and SPR. ${ }^{5}$ Finally, since the transducer is 23 separated from the sensing surface, the EGFET configuration readily enables the integration of 24 potentiometric sensors with other sensor platforms. 
1 EIS-based impedimetric biosensors measure change of impedance when species attach to the sensing

2 surface. Under faradaic operation involving redox couples, the measured impedance spectrum can be

3 further analyzed using an equivalent circuit model. Two of the most important parameters, charge transfer

4 resistance $\left(R_{\mathrm{ct}}\right)$ and surface capacitance $\left(C_{\text {surface }}\right)$ can be resolved in this way. The change of surface

5 potential and the formation of the biomolecular layer will prevent the redox species from approaching the

6 working electrode due to electrostatic repulsion and steric hindrance, respectively. ${ }^{7}$ The phenomenon is

7 often reflected in an increase of the $R_{\mathrm{ct}}$ which dominates the overall measured impedance.

8 In this letter, we demonstrate a fully integrated system that combines both two-terminal EGFET-based

9 potentiometric and three-terminal EIS-based impedance biosensing on a shared active sensing surface.

10 This system enables the sequential measurement of the same binding event on the same sensing surface

11 using two different sensing techniques. Consequently, we are able to perform a fundamental investigation

12 into the origins of the sensing signal produced by FET and EIS biosensors. A bovine serum albumin

13 (BSA) versus antibody-BSA (anti-BSA) prototype system was used as the test vehicle for the integrated

14 biosensors. In addition, detection of bovine parainfluenza antibodies in a complex blood system with

15 hemagglutinin-neuraminidase (HN) has also been demonstrated. Comparison of the EGFET and EIS

16 sensor responses reveals similar dynamic ranges, and motivates our study of the relationship between

17 surface potential and impedance, which is explained by electrochemical kinetics and the Butler-Volmer

18 equation.

19 All the chemicals and proteins were purchased from Sigma-Aldrich and used as received. The sensor chip

20 was fabricated on a borofloat wafer. The metallic structures including Au micro-strip working electrode

21 (WE), Au (Ti/Au 10/90 nm) counter electrode (CE) and Pt (Ti/Pt 10/90 nm) on-chip pseudo-reference

22 electrode (RE) were deposited and patterned using e-beam evaporation and photolithography, respectively.

23 A commercial n-MOSFET (VN0104, Supertex) was used as the transducer for EGFET biosensors and the

24 I-V characterization was measured using a Keithley 4200-SCS semiconductor analyzer. EIS biosensors

25 were characterized using a Gamry Interface 1000 potentiostat. The WE was coated with o-aminobenzoic 
acid (o-ABA, $50 \mathrm{mM}$ in $\mathrm{H}_{2} \mathrm{SO}_{4}$ ) carboxylated film through 10 cyclic voltammetry scans (0 to $0.8 \mathrm{~V}, 50$

$2 \mathrm{mV} / \mathrm{s}$ ). The BSA proteins (1\% in PBS) were immobilized onto the sensing surface through amine

3 coupling using NHS/EDC. The contact time for BSA protein immobilization is 30 minutes. The sensing

4 surface was later deactivated and blocked using $1 \mathrm{M}$ ethanolamine- $\mathrm{HCl}$ solution for 30 minutes. This

5 sensing surface was exposed to anti-BSA dilutions in PBS with 20 min contact time. The detailed

6 experimental methods of HN/BPIV3 sensing can be found in the Supplementary Information. A $10 \mathrm{mM}$

7 hexaammineruthenium (III) chloride in PBS was used as the redox couple. In the EGFET sensor, the Au

8 active sensing surface serves as the WE, i.e. extended gate of a MOSFET. A DC voltage sweep is applied

9 to the liquid through a commercial $\mathrm{Ag} / \mathrm{AgCl} \mathrm{RE}$. For EIS sensor, the aforementioned $\mathrm{Au}$ WE is the active

10 sensing surface. On-chip Pt pseudo-RE and Au CE are used. The signal, i.e. surface potential, of

11 potentiometric sensors was extracted from the $I_{\mathrm{d}}-V_{\text {ref }}$ curve as the $V_{\text {ref }}$ value when $I_{\mathrm{d}}=1 \mu \mathrm{A}$. The EIS

12 spectra were modeled using ZView (Scribner Associates Inc.) by minimizing the chi square value. The

13 detailed methods and parameters for the electrical measurement can be found in Supplementary

14 Information.

15 Comparing EGFET and EIS sensors, the Au working electrode plays a common role: an active sensing 16 surface and WE. As a result, a fully integrated sensing system combining EGFET potentiometric and EIS

17 impedance biosensors can be achieved by sharing a common Au WE as schematically shown in Figure 1a.

18 Using this integrated system, the steps of chemical functionalization and biomolecular attachment can be 19 achieved for both sensors concurrently. Furthermore, this design offers a direct comparison between

20 potentiometric and impedance sensors because the same binding events will be measured on a shared

21 sensing surface.

22 The optical image of the sensor chip is shown in Figure 1c and the enlarged view of Au micro-strip WE in

23 Figure 1d. The dimension of the Au micro-strip is $50 \mu \mathrm{m}$ long and $0.8 \mu \mathrm{m}$ wide $\left(4 \times 10^{-7} \mathrm{~cm}^{2}\right)$. The use of a

24 microscale electrode provides the benefit of minimizing the mass transfer limited behaviors in EIS at low

25 frequency, due to the decreased diffusion length around the miniaturized working electrode. ${ }^{9,14,15}$ The 
conventional Randle circuit model comprises of series resistance $\left(R_{\mathrm{s}}\right)$, surface capacitance $\left(C_{\text {surface }}\right.$ usually

2 modeled using constant phase elements, CPE), Warburg resistance (W) and charge transfer resistance

$3\left(R_{\mathrm{ct}}\right)$. When the nominal size of the working electrode decreases, the diffusion layer thickness decreases,

4 and the current is less dependent on the diffusion of reacting species toward the WE. ${ }^{16}$ As a result, the

5 mass transfer dominated $\mathrm{W}$ becomes negligible and the Randle circuit can be simplified to a simple RC

6 circuit as shown in Figure 1b. ${ }^{17,18}$ The measured impedance spectra will be modeled using the simplified

7 circuit model to extract the circuit elements, including $R_{\mathrm{ct}}$. Using an n-channel MOSFET as the transducer,

8 a positive voltage is applied to the solution for FET biosensor measurement and, therefore, a redox probe

9 that has negative redox potential is desired. As a result, hexaammineruthenium (III) chloride, which has

10 both negative reduction and oxidation potentials, qualifies for the application in this study.

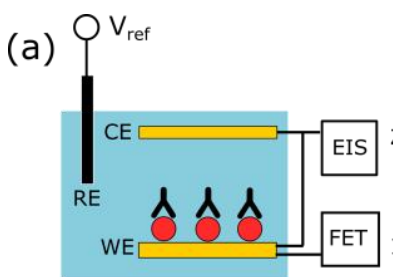

\section{(b)}
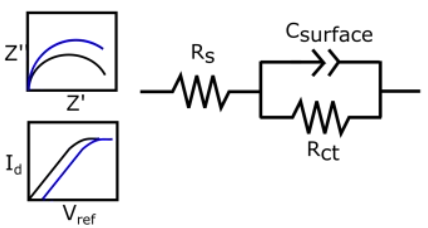

\section{(c)}
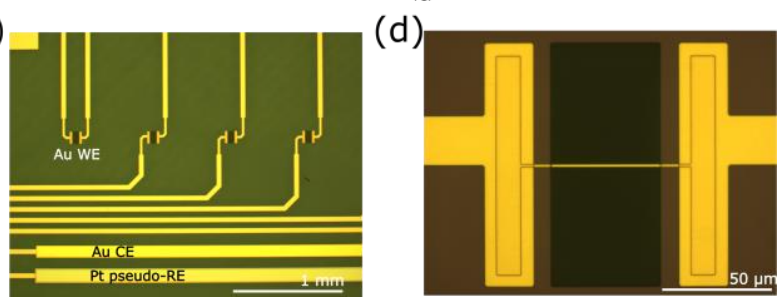

13 Figure 1. The scheme and the optical image of the integrated FET/EIS biosensor system. (a) The experimental setup of the integrated system. (b) The equivalent circuit model for the impedance spectroscopy. (c) - (d) The optical images of the biosensor chip and the enlarged view of the Au microstrip working electrode.

The FET/EIS integrated sensor's responses to the BSA versus anti-BSA system are shown in Figure 2.

The $I_{\mathrm{d}}-V_{\text {ref }}$ curve of the transistor will shift horizontally depending on the magnitude and the polarity of 
1 the change of surface potential on the Au sensing surface. This change of surface potential due to the

2 attachment of charged antibodies to the WE, i.e. extended gate, was obtained using the change of the

3 FET's threshold voltage $\left(V_{\mathrm{th}}\right)$. Figure 2a depicts the shift of $I_{\mathrm{d}^{-}} V_{\text {ref }}$ curves of the potentiometric sensor in

4 the positive direction in response to the increasing concentration of anti-BSA in the PBS, indicating that

5 the anti-BSA (isoelectric point $4.8-5.2$ ) is negatively charged in the PBS buffer (pH 7.4). ${ }^{19}$ Figure $2 \mathrm{~b}$

6 shows the change of surface potential measured by FET potentiometric sensor versus anti-BSA

7 concentration in linear scale and logarithmic scale (inset of Figure 2b). A typical Langmuir adsorption

8 response with a gradual saturation toward high antibody concentration was observed. ${ }^{4}$ Measuring the

9 same binding event at the working electrode, the change of EIS spectra with respect to anti-BSA

10 concentration is presented in a Nyquist plot (Figure 2c). Taking advantage of the miniaturized working

11 electrode, no obvious mass transfer limited characteristics were observed in the recorded impedance

12 spectra, except a slight deviation from the semi-circle under low frequency $(<5 \mathrm{~Hz})$ and a further

13 optimization of the WE may be needed. As a result, the change of surface properties on the sensing

14 surface is mainly reflected through the change of $R_{\mathrm{ct}}$. As the anti-BSA concentration increases, the overall

15 size of the semicircle increases, indicating an increase of $R_{\mathrm{ct}}$ and total impedance. By fitting the

16 impedance spectra with the equivalent circuit model (Figure 1b), biomolecular binding-related circuit

17 elements, $R_{\mathrm{ct}}$ and $C_{\text {surface, }}$, could be extracted. However, $C_{\text {surface }}$ is relatively insensitive to the attachment of

18 antibodies to the semipermeable biolayer (Figure S1). Figure $2 \mathrm{~d}$ shows the relationship between $R_{\mathrm{ct}}$ and

19 anti-BSA concentration. A similar Langmuir adsorption behavior with saturation at high anti-BSA

20 concentration was again observed, showing integrated operation of a FET/EIS biosensor system.

21 Furthermore, the similar response obtained from both sensing platforms suggests a possible correlation

22 between surface potential and the impedance, which is explored in the following. 

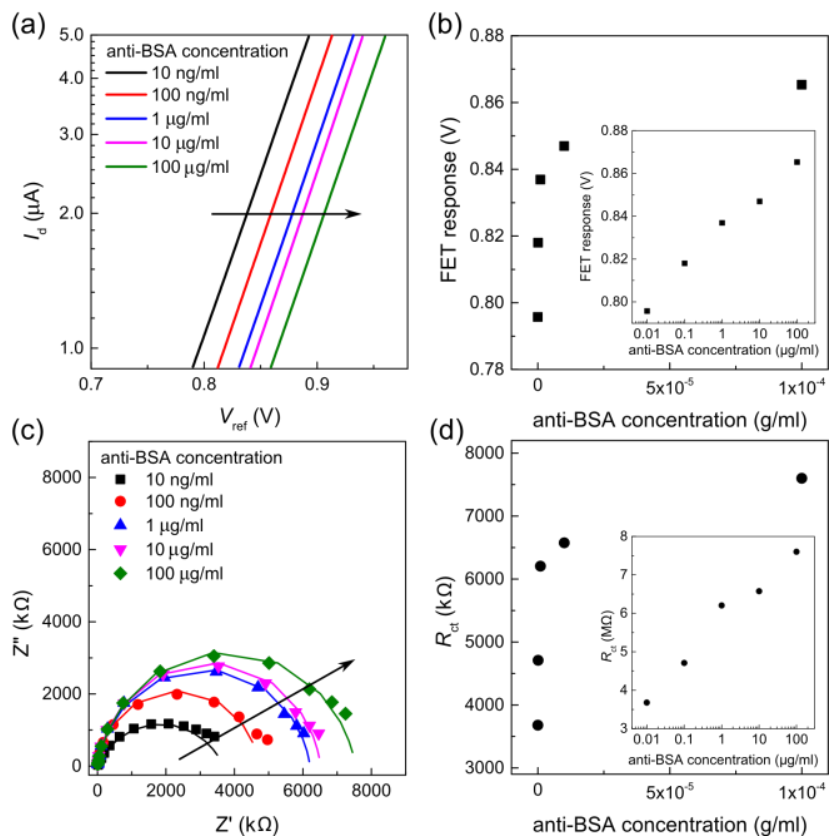

Figure 2.The responses of FET potentiometric and EIS impedance biosensors to anti-BSA concentrations. (a) The shift of $I_{\mathrm{d}}-V_{\text {ref }}$ curve measured with FET and (b) the FET signal (surface potential) in response to anti-BSA concentrations in linear scale and logarithmic scale (inset). (c) Nyquist plots of impedance spectra measured with EIS and (d) the $R_{\mathrm{ct}}$ change in response to anti-BSA concentrations in linear scale and logarithmic scale (inset).

According to classical electrochemical kinetics, the electrical current that flows through the electrode is governed by the effective potential on the electrode surface. ${ }^{20}$ This relationship is expressed by the ButlerVolmer equation. Under bias conditions that deviate from equilibrium, i.e. Tafel behavior, the ButlerVolmer equation that links the charge transfer resistance and the surface potential can be simplified to: ${ }^{20,21}$

$12 R_{c t}=R_{0} \exp \left(\frac{\alpha q \Delta V}{k_{B} T}\right)$ where $R_{0}=E_{\text {app }} / F A K^{0} C, F$ is Faraday constant, $A$ is the area of the working electrode, $k^{0}$ is the standard rate constant, $C$ is the concentration of the redox couple species at the electrolyte-electrode interface, transfer coefficient $\alpha$ is commonly assumed to be 0.5 for a symmetrical energy barrier, $E_{\text {app }}$ is the bias applied to the system, $\Delta V$ is the change of surface potential on the working electrode, $q$ is the elementary 
charge of an electron, $k_{\mathrm{B}}$ is Boltzmann's constant and $T$ is the temperature. It is reported that $k^{0}$ exhibits a

2 wide range from $10^{-9}$ to several hundred $\mathrm{cm} / \mathrm{s}$ depending on the choice of the redox couple, the surface

3 morphology of the WE, the formation of the biolayer, different charge transfer mechanisms, buffer

4 concentration, etc. ${ }^{20,22,23}$ In effect, the Butler-Volmer equation predicts that $R_{\mathrm{ct}}$ is exponentially related to

5 the change of surface potential $(\Delta V)$. Figure 3 shows the relationship between $R_{\mathrm{ct}}$, measured with EIS, and

6 the exponentiation of $\Delta V$, i.e. $\exp \left(\alpha q \Delta V / k_{\mathrm{B}} T\right)$, obtained from the FET. A high linearity is observed which

7 agrees well with the prediction of Butler-Volmer theory. The fitted $R_{0}$ is $1.43 \times 10^{6} \Omega$ and is comparable

8 to the calculated value of $1.04 \times 10^{6} \Omega$, where $A=4 \times 10^{-7} \mathrm{~cm}^{2}, k^{0}$ is $1 \mathrm{~cm} / \mathrm{s}$ for a simple electron

9 transfer, ${ }^{23}$ and $C$ is $10^{-5} \mathrm{~mol} / \mathrm{cm}^{3}$. In the case of affinity sensors, the $\Delta V$ results from the binding of

10 charged biomolecules to the surface, i.e. WE. EGFET sensors directly measure this surface potential

11 change, which concurrently influences the electrical current and the resulting impedance measured by EIS

12 sensors by increasing the hindrance for redox species from reaching the WE. This finding confirms that

13 the origin of the sensing signals for both potentiometric biosensors and impedance biosensors is the

14 change of surface potential. Also, the signal output from potentiometric biosensors can be linked to that of impedance biosensors using the Butler-Volmer equation.

16 Beyond the BSA/anti-BSA system, a complex serological system of bovine hemagglutinin-neuraminidase

$17(\mathrm{HN})$ versus antibodies of bovine parainfluenza virus protein type-3 (BPIV3) in blood plasma samples

18 was also tested. ${ }^{24} \mathrm{HN}$ protein was immobilized on the shared WE via amine coupling as the probe against the BPIV3 proteins in the analyte solution. Similar to BSA/aBSA system, similar trends of the dynamic range of FET and EIS sensors were observed. Again, the relationship between signals from potentiometric and impedance sensors, $\Delta V$ and $R_{\mathrm{ct}}$, respectively, are linked and agrees well with Butler-Volmer theory

22 (see Supplementary Information and Figure S2). The results further confirm the correlation between

23 potentiometric and impedance sensors and suggest that the electrical sensor is a promising tool for the 24 disease diagnostic applications. The details of HN protein immobilization, sensing surface blocking and 25 detection of antibodies of BPIV3 can be found in Supplementary Information. 


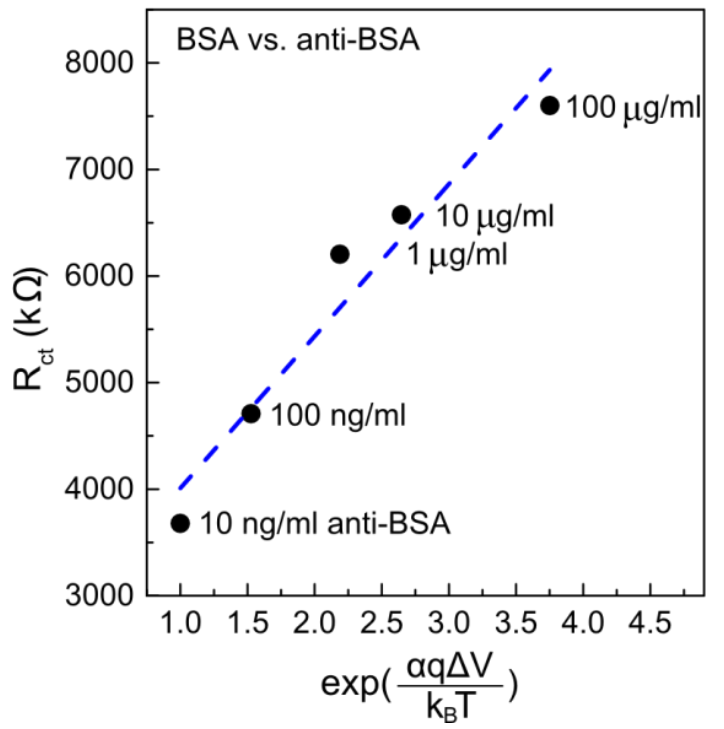

2 Figure 3. The relationship between the exponentiation of change of surface potential $\left(\exp \left(\alpha q \Delta V / k_{\mathrm{B}} T\right)\right)$ 3 and the charge transfer resistance $\left(R_{\mathrm{ct}}\right)$, measured with potentiometric and impedemetric biosensors, 4 respectively. The linear relationship fits well into Butler-Volmer equation and confirms that both 5 potentiometric and impedance biosensors are charge sensitive.

Impedance spectroscopy is a powerful tool to analyze the interfacial properties for corrosion, coatings,

8 batteries, solar cells, etc. through complex analysis of the frequency responses. ${ }^{25-29}$ Intuitively, surface

9 impedance measured in EIS biosensors is expected to respond to both charge and mass of the

10 biomolecules. However, for biosensor applications, the frequency response of surface capacitance is

11 insensitive to the formation of the biomolecular layer on the sensing surface because: (1) the biolayer is

12 semipermeable and is not a good capacitor; and, (2) the double layer capacitance dominates. ${ }^{67,18}$ As a

13 result, it is difficult to resolve the mass-related properties of biomolecules alongside their charge

14 information using impedance biosensors. The surface potential related $R_{\mathrm{ct}}$ remains the dominating

15 contribution for the sensing signal. In other words, the FET/EIS integrated sensing system provides little

16 extra value in monitoring the charge information of biomolecules since the origin of the signal is linked. 
1 In this way, simple FET-based potentiometric biosensors provide sufficient information for practical

2 biosensing applications.

3 To further illustrate their suitability for cost-effective and rapid point-of-care detection, large area $\mathrm{Au}$

4 extended gate sensor chips $\left(0.1 \mathrm{~cm}^{2}\right)$ were also prepared without the use of photolithography steps,

5 instead using e-beam metal evaporation and patterning with shadow masks. The DC operation of the

6 EGFET at steady state is not limited by the diffusion and, therefore, alleviates the constraints of using

7 microscale electrodes. The BSA proteins were immobilized onto widely used thiol SAM through amine

8 coupling. The sensor response of the large area Au chips is compared to the micro-strip sensors in Figure

9 4. In both cases, the BSA/anti-BSA sensitivities are around $17-18 \mathrm{mV} /$ decade change of anti-BSA

10 concentration. The results indicate no sensitivity advantage to the micro-strip design in EGFET biosensor

11 at steady state.

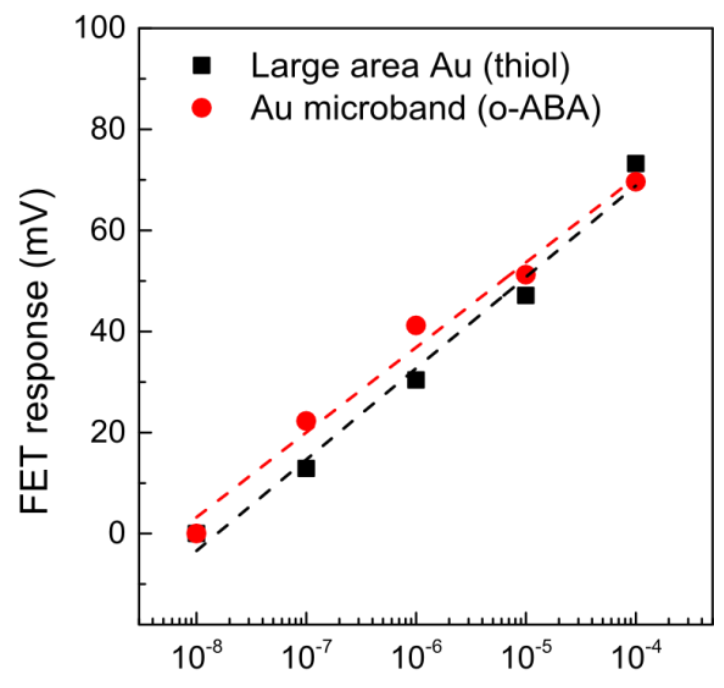

anti-BSA concentration $(\mathrm{g} / \mathrm{ml})$

13 Figure 4. The comparison between EGFET biosensors using large area Au sensing surface (black square) 14 and Au micro-strip (red circle). The BSA/anti-BSA sensitivities using either approach are comparable. 
1 In conclusion, biosensing using an integrated two-sensor system that combines EGFET-based

2 potentiometric biosensors and EIS-based impedimetric biosensors has been demonstrated. This integrated

3 two-sensor system enables the direct comparison between two sensing techniques by monitoring the same

4 biological layers on the sensing surface. The correlation between sensor signals - that is, surface potential

5 and $R_{\mathrm{ct}}$ from FET and EIS, respectively - was linked using the Butler-Volmer equation. The results

6 suggest that both FET and EIS biosensors are sensitive to the change of surface potential due to the

7 attachment of charged biomolecules to the sensing surface, whereas the mass-dependent value of $C_{\text {surface }}$

8 does not vary significantly. For the detection of charge properties of biomolecules, the EIS/FET

9 integrated sensing system provides little extra advantage. Therefore, EGFET biosensors are inherently

10 sufficient as electrical transducers for label-free biomolecule detection, while providing the added

11 benefits of rapid response and low-cost scalability. For scenarios where detailed information about both

12 charge and mass properties of biomolecular targets is required, the FET/EIS integrated sensing system

13 that we have proposed may be deployed to great effect. Future studies involving complex biofluids and

14 advanced modeling will help to identify how the mass-related properties extracted from EIS can be 15 leveraged.

\section{Supplementary Material}

18 The Supplementary Materials include (1) details of electrical measurement and the integrated sensor

19 operation; (2) the extracted capacitance response against different anti-BSA concentrations from EIS

20 spectra; (3) the detailed description and results of HN/BPIV3 animal disease sensing. 
This work was supported by: the National Science Foundation (NSF, through CBET Award \#1264705);

the Science Foundation Ireland under US-Ireland "Agri-Sense" project (SFI12/US/I2476); the

\section{References}

M. L. Y. Sin, K. E. Mach, P. K. Wong, and J. C. Liao, Expert Rev. Mol. Diagn. 14 (2), 225 (2014). P. Bergveld, IEEE Trans. Biomed. Eng 17 (1), 70 (1970).

3 M. S. Ozdemir, M. Marczak, H. Bohets, K. Bonroy, D. Roymans, L. Stuyver, K. Vanhoutte, M. Pawlak, and E. Bakker, Anal. Chem. 85 (9), 4770 (2013).

4 A. Tarasov, M.-Y. Tsai, E. M. Flynn, C. A. Joiner, R. C. Taylor, and E. M. Vogel, 2D Mater. 2 (4), 044008 (2015).

5 A. Tarasov, D. W. Gray, M.-Y. Tsai, N. Shields, A. Montrose, N. Creedon, P. Lovera, A. O'Riordan, M. H. Mooney, and E. M. Vogel, Biosens. Bioelectron. 79, 669 (2016).

$6 \quad$ M. I. Prodromidis, Electrochim. Acta 55 (14), 4227 (2010).

7 J. S. Daniels and N. Pourmand, Electroanalysis 19 (12), 1239 (2007).

$8 \quad$ E. Katz and I. Willner, Electroanalysis 15 (11), 913 (2003).

9 A. Montrose, N. Creedon, R. Sayers, S. Barry, and A. O'Riordan, Journal of Biosensors \& Bioelectronics 6 (3), 174 (2015).

10 M. O. Noor and U. J. Krull, Anal. Chim. Acta 825, 1 (2014).

11 B. Zhan, C. Li, J. Yang, G. Jenkins, W. Huang, and X. Dong, Small 10 (20), 4042 (2014).

12 X. Tang, S. Bansaruntip, N. Nakayama, E. Yenilmez, Y.-1. Chang, and Q. Wang, Nano Lett. 6 (8), 1632 (2006).

13 P. Dak, P. Nair, G. Jonghyun, and M. A. Alam, presented at the Device Research Conference (DRC), 2013 71st Annual, 2013.

14 A. Wahl and A. O'Riordan, in Electrochemical Strategies in Detection Science (The Royal Society of Chemistry, 2016), pp. 205.

15 A. Wahl, S. Barry, K. Dawson, J. MacHale, A. J. Quinn, and A. O'Riordan, J. Electrochem. Soc. 161 (2), B3055 (2014).

16 M. J. Madou and R. Cubicciotti, Proc. IEEE 91 (6), 830 (2003).

17 W. Franks, I. Schenker, P. Schmutz, and A. Hierlemann, IEEE Trans. Biomed. Eng 52 (7), 1295 (2005).

18 R. Radhakrishnan, I. I. Suni, C. S. Bever, and B. D. Hammock, ACS Sustain Chem Eng 2 (7), 1649 (2014).

19 C. Wang, J. Wang, and L. Deng, Nanoscale Res. Lett. 6 (1), 579 (2011).

20 A. J. Bard and L. R. Faulkner, Electrochemical Methods: Fundamentals and Applications, 2 ed. (John Wiley \& Sons, Inc., 2001).

21 A. ter Heijne, O. Schaetzle, S. Gimenez, L. Navarro, B. Hamelers, and F. Fabregat-Santiago, Bioelectrochemistry 106, Part A, 64 (2015).

H. V. M. Hamelers, A. ter Heijne, N. Stein, R. A. Rozendal, and C. J. N. Buisman, Bioresour. Technol. 102 (1), 381 (2011). 
H. O. Finklea, L. Liu, M. S. Ravenscroft, and S. Punturi, J. Phys. Chem. 100 (48), 18852 (1996).

24 D. W. Gray, M. D. Welsh, S. Doherty, F. Mansoor, O. P. Chevallier, C. T. Elliott, and M. H. Mooney, Vet Res. 46 (1), 7 (2015).

25 A. Lasia, Electrochemical Impedance Spectroscopy and its Applications. (Springer-Verlag New York, 2014), p.367.

26 N. A. Cañas, K. Hirose, B. Pascucci, N. Wagner, K. A. Friedrich, and R. Hiesgen, Electrochim. Acta 97, 42 (2013).

F. Mansfeld, J. Appl. Electrochem. 25 (3), 187 (1995).

M.-Y. Tsai, W.-H. Cheng, J.-S. Jeng, and J.-S. Chen, Solid-State Electron. 120, 56 (2016).

W.-H. Cheng, J.-W. Chiou, M.-Y. Tsai, J.-S. Jeng, J.-S. Chen, S. L.-C. Hsu, and W.-Y. Chou, J. Phys. Chem. C 120 (28), 15035 (2016). 